\title{
COMMUNITY ENGAGEMENT FOR Wolbachia-BASED Aedes aegypti POPULATION SUPPRESSION FOR DENGUE CONTROL: THE SINGAPORE EXPERIENCE
}

\author{
C. LIEW, L. T. SOH, I. CHEN, X. LI, S. SIM AND L. C. NG \\ Environmental Health Institute, National Environment Agency, Singapore; \\ NG_Lee_Ching@nea.gov.sg
}

\begin{abstract}
SUMMARY
Despite a long-running and comprehensive national dengue control programme, Singapore continues to experience regular outbreaks of dengue. The Environmental Health Institute of the Singapore National Environment Agency (NEA) is thus evaluating a Wolbachia-based combined Incompatible and Sterile Insect Technique approach (IIT/SIT) as a dengue control strategy. This approach involves field releases of irradiated male Wolbachia-carrying Aedes aegypti (L.) mosquitoes, with the aim of further suppressing the urban Aedes aegypti mosquito population and reducing dengue transmission. Since the beginning of our project, we considered community education and engagement as a key factor for the success of the field studies. We have therefore conducted extensive groundwork to share and consult with, and engage a wide range of stakeholders, including residents at the study sites, the general public, the medical and scientific communities, and government agencies. In this chapter, we outline our strategy for engaging residents at the study sites and the general public (two primary stakeholder groups), focusing on the key principles around which we have built our approach.
\end{abstract}

Key Words: Mosquitoes, Aedes albopictus, Wolbachia, cytoplasmic incompatibility, Incompatible Insect Technique, Sterile Insect Technique, outreach, community education, public mobilisation, high-rise urban environment, public housing apartments

\section{INTRODUCTION}

Alongside rapid urbanisation and increased global travel, the worldwide incidence of dengue has risen dramatically in recent decades. Today, around 3900 million people in 128 countries are at risk of contracting the disease, with an estimated 390 million annual infections worldwide (Bhatt et al. 2013).

In Singapore, the four serotypes of dengue virus are transmitted between humans, mainly by Aedes aegypti (L.), the yellow fever mosquito, with Aedes albopictus (Skuse), the Asian tiger mosquito, as a secondary vector. Reflecting the global situation, dengue transmission in Singapore correlates with the presence of Ae. 
aegypti, whilst areas with Ae. albopictus alone are associated only with isolated dengue cases (Hapuarachchi et al. 2016; Ong et al. 2019).

Singapore's long-running and comprehensive dengue management programme, which has a strong focus on source reduction and environmental management, now maintains the Aedes House Index (the percentage of properties in which breeding sites are detected, Ong et al. 2019) at low levels of around 2\%. Yet, despite effective suppression of the Aedes mosquito vector populations, Singapore continues to experience regular dengue outbreaks, with explosive ones - associated with switching of the predominant dengue virus serotype (Lee et al. 2010) - occurring in 2005, 2007, and 2013-2014.

The continued susceptibility of Singapore's population to dengue outbreaks may be attributed to multiple factors, including: a highly urbanised, high-density, and highrise environment; reduced herd immunity after decades of low local dengue transmission; transmission outside homes, such as at schools and workplaces; and the presence of cryptic (or unusual) Aedes mosquito breeding sites that are difficult to detect (Dieng et al. 2012; Low et al. 2015), amongst others. These challenges, coupled with the absence of approved antivirals and an effective vaccine against dengue, highlight the urgent need for novel and sustainable vector and dengue control approaches.

One new approach, currently being tested in field trials in Asia, the Americas, Australia, and the Pacific islands, involves the release of Ae. aegypti mosquitoes carrying the Wolbachia bacterium (McMeniman et al. 2009). In Singapore, the Environmental Health Institute (EHI) of the National Environment Agency (NEA) is trialling since 2016 a Wolbachia-based combined Incompatible and Sterile Insect Technique (IIT/SIT) approach, involving releases of irradiated male Wolbachiacarrying Ae. aegypti (Wolbachia-Aedes) mosquitoes (Lees et al. 2015; Bourtzis et al. 2016; Dyck et al. 2021). As the eggs resulting from mating between released male Wolbachia-Aedes mosquitoes and female urban Ae. aegypti mosquitoes are nonviable, the initiative, termed 'Project Wolbachia - Singapore', ultimately aims to further suppress mosquito populations and hence the risk of dengue transmission (Liew and Ng 2019; NEA 2019a). Irradiation is used to sterilise the small percentage of female Wolbachia-Aedes mosquitoes remaining after sorting mass-reared pupae, thus preventing population replacement stemming from their release (Bourtzis et al. 2016; Lees et al. 2021).

Project Wolbachia - Singapore's IIT/SIT approach aligns well with Singapore's long-standing emphasis on mosquito population suppression, and is intended to complement traditional vector control measures, such as mosquito breeding habitat removal, space spraying with insecticides, and entomological surveillance. Existing Singapore legislation provides for NEA to produce Wolbachia-Aedes mosquitoes for research purposes, and the approval for releases of these male mosquitoes was obtained at the ministerial level.

Singapore has a high human population density of almost 8000 people per $\mathrm{km}^{2}$, and more than $80 \%$ of the population lives in public housing apartments (SingStat 2019). This makes Project Wolbachia - Singapore the first trial in the world to use the technology in such a high-rise, high-density urban environment. 
In addition to rigorous laboratory studies and risk assessment, community engagement has been an integral component of Project Wolbachia - Singapore since its inception. Since 2012 (well before field releases commenced in 2016), EHI has carried out extensive groundwork to share and to consult with, and engage stakeholders, including residents at the study sites, the general public, the medical and scientific communities, schools and tertiary institutions, and government agencies.

These efforts continue today, as Project Wolbachia - Singapore trials advance in stage and scope. As of December 2019, trial releases accompanied by community engagement activities have been conducted in two study sites of 163 and 121 highrise apartment blocks respectively (Fig. 1), covering a total of 27600 households. Here, we present an overview of our strategies for dialoguing with residents at the study sites, and the public, with an emphasis on the key community engagement principles that have guided our approach.

\section{PROJECT WOLBACHIA - SINGAPORE COMMUNITY ENGAGEMENT PRINCIPLES}

Community engagement is essential to the testing and future use of novel vector control technologies involving the release of modified mosquitoes. While there are differences in country contexts and technologies used, studies on the design, implementation and evaluation of such community engagement programmes emphasise common principles, such as inclusive public engagement and consultation, transparency, and tailoring the engagement to the local audience (Subramaniam et al. 2012; McNaughton and Duong 2014; Ernst et al. 2015; Kolopack et al. 2015).

To achieve effective and respectful outreach for Project Wolbachia - Singapore, we developed a framework for engaging residents and the public based on the principles laid out in this Section. This framework also builds upon existing professional collaborations in research and dengue management, as well as longstanding outreach efforts urging the general public to remove and destroy Aedes mosquito breeding habitats in their homes (NEA 2019b).

Whilst this chapter focuses on residents at the study sites and the general public, it should be noted that our community engagement efforts also extend to other stakeholder groups. For example, we conduct scientific seminars, lectures, and workshops to inform and consult the scientific and medical communities about Wolbachia-Aedes technology. We additionally consult with international experts to share knowledge and key findings, and also hold seminars and workshops to engage stakeholders in the government, including agencies with functions in public health and the environment. The feedback received from all stakeholder groups has been instrumental in shaping our outreach and deployment strategies. 

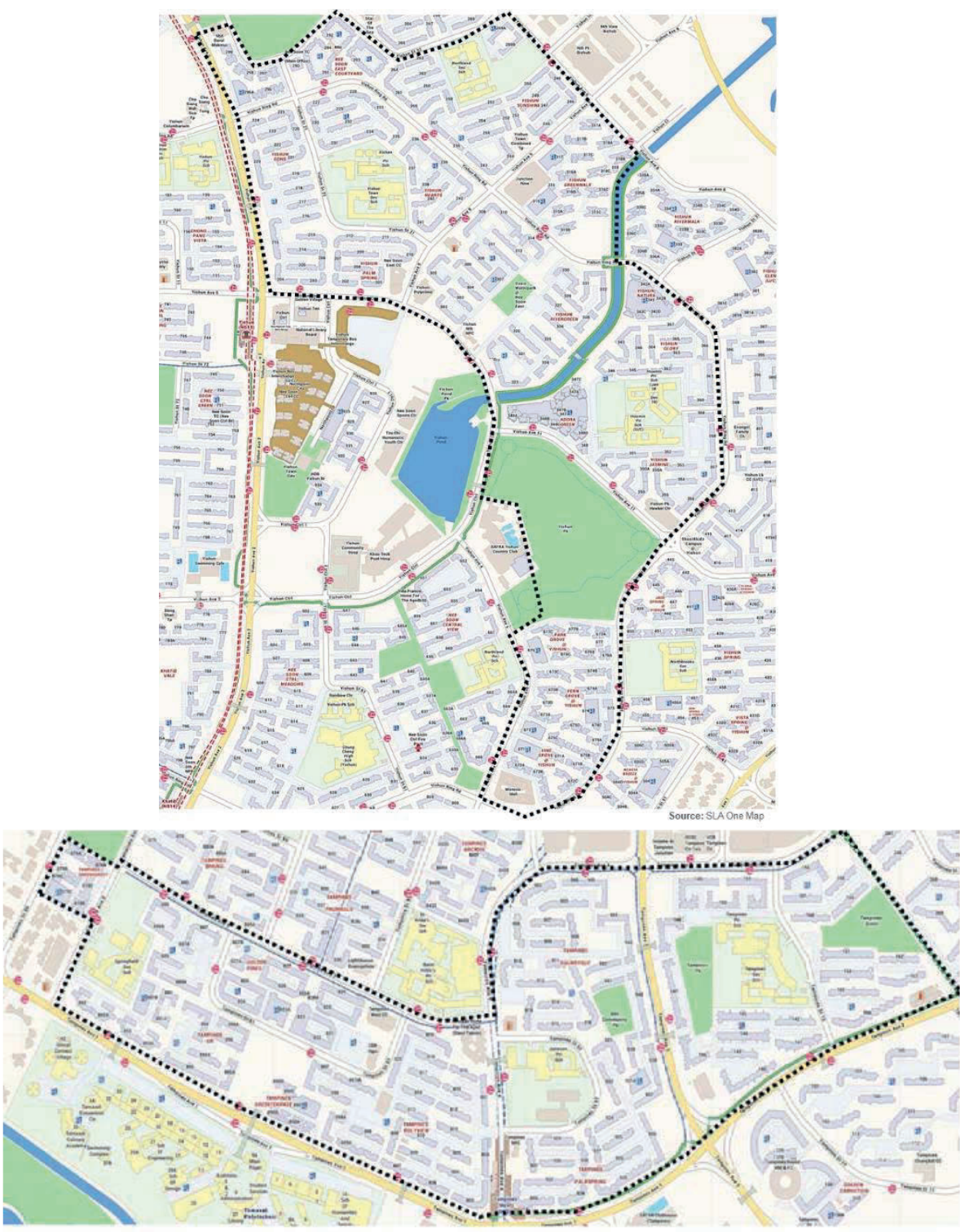

Figure 1. Project Wolbachia - Singapore study sites as of December 2019. Trial releases of male Wolbachia-carrying Aedes aegypti mosquitoes, accompanied by community engagement activities, have been conducted in 163 high-rise apartment blocks at Nee Soon East (top) and 121 at Tampines West (bottom), covering a total of 27600 households. 


\subsection{Transparent, Clear and Consistent}

Project Wolbachia - Singapore involves the release of male Wolbachia-Aedes mosquitoes in close proximity to residential dwellings. We thus endeavoured to make our community engagement as accurate and clear as possible, so that residents and the public are well informed about the intervention taking place in their neighbourhoods and the possible effects that this may have on their households.

Importantly, our community engagement got off to an early start. We initiated outreach activities in 2012, in parallel with our laboratory studies and risk assessment of the Wolbachia-Aedes technology, well before the first male Wolbachia-Aedes mosquitoes were released in 2016. This allowed time for members of the public to familiarise themselves with Aedes mosquito biology and behaviour, such as the fact that male mosquitoes do not bite, and with Wolbachia-Aedes technology in general. We also had sufficient time to address potential issues raised by stakeholders; for example, we conducted laboratory studies to confirm that male Wolbachia-Aedes mosquitoes do not lose the Wolbachia bacterium as they age, a concern that was raised by an expert entomologist.

Given that public messaging in Singapore has for decades emphasised the importance of keeping mosquito populations low, the proposed strategy of releasing large numbers of male Wolbachia-Aedes mosquitoes may be seen by some as counterintuitive. Thus, we sought to ensure that Project Wolbachia - Singapore's purpose and goals - that the mosquito releases, together with existing control methods being applied, are compatible and intended to further suppress mosquito populations-were clearly communicated to the public.

To avoid conflicting messaging, consistency is also key. For example, residents at the release sites are encouraged to remain vigilant and continue practising standard mosquito control activities, such as turning over pails and flowerpots, and clearing roof gutters. Residents are also advised to kill adult mosquitoes as they normally would.

As Project Wolbachia - Singapore scaled up and progressed to more advanced stages (NEA 2018a, 2019c), we also delivered prompt updates to keep the public abreast of new developments. These developments included the releases of male Wolbachia-Aedes mosquitoes at higher floors, in addition to the releases at the ground floors; collaborations with private sector companies to incorporate technologies such as automated devices for mosquito production, sorting, and release (NEA 2018b); and the use of irradiation post-pupal sorting to sterilise any female Wolbachia-Aedes mosquitoes remaining from the rearing process among the males to be released (NEA 2018a).

We have endeavoured to provide members of the public with adequate information, so that they can develop informed opinions on the risks and benefits of Project Wolbachia - Singapore. At the same time, we also sought to present this information in a manner that is accessible to individuals without scientific training. Striking this balance is important so that key messages are not obscured by technical details and scientific jargon yet remain accurate. 


\subsection{Science-based and Educational}

Misinformation is a common source of fear and doubt surrounding new technologies. Our community engagement thus aims to demystify the Wolbachia-Aedes technology, by equipping the public with a strong understanding of the scientific concepts behind it.

In our outreach materials and engagement sessions, we use accessible language and infographics to explain concepts such as the origin and natural occurrence of the Wolbachia bacterium, cytoplasmic incompatibility, and how the release of male Wolbachia-Aedes mosquitoes will lead to a reduction in Ae. aegypti mosquito populations. Project Wolbachia - Singapore scientists and technicians were also heavily involved in outreach and were often on-site during door-to-door house visits, dialogue/outreach sessions and roadshows, to answer any questions related to the technology.

Where possible, we also created hands-on experiences for the public to learn about Wolbachia-Aedes technology. For example, participants at our roadshows and dialogue/ outreach sessions were encouraged to place their hands into transparent Perspex boxes containing male Wolbachia-Aedes mosquitoes, so that they could experience for themselves that male mosquitoes do not bite. We also organise regular tours of NEA's Wolbachia-Aedes mosquito production facility (Fig. 2), where members of the public learn how mosquitoes are reared, sorted, and prepared for release.

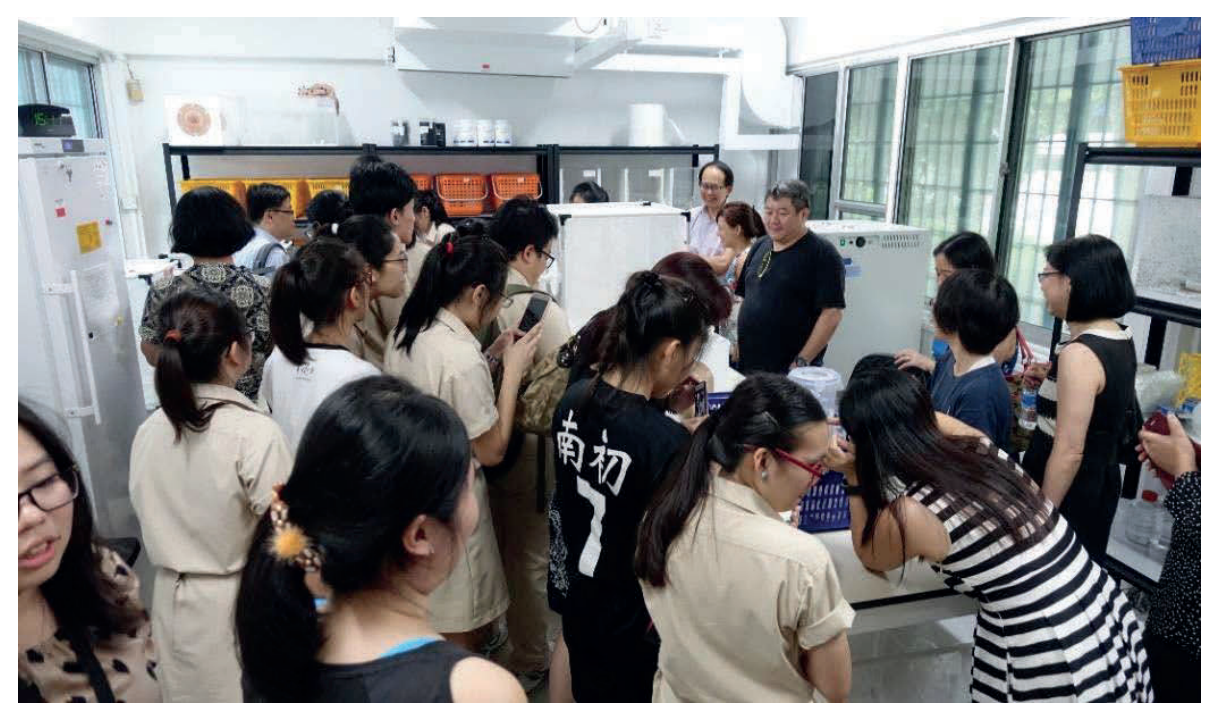

Figure 2. Environmental Health Institute (EHI) researchers conducting a tour of the National Environment Agency's (NEA's) Wolbachia-Aedes mosquito production facility for members of the public, during a Project Wolbachia - Singapore learning journey. 
As children and the youth community are often readily engaged and may also proactively help convey information to their families, we also hold sharing sessions at schools and tertiary institutions. Additionally, we have been engaging childcare centres (Fig. 3), and have been working with schools located within the release sites, to provide hands-on lessons related to Project Wolbachia - Singapore, including the release of male Wolbachia-Aedes pupae around school premises, and the identification of mosquitoes trapped by Gravitraps.

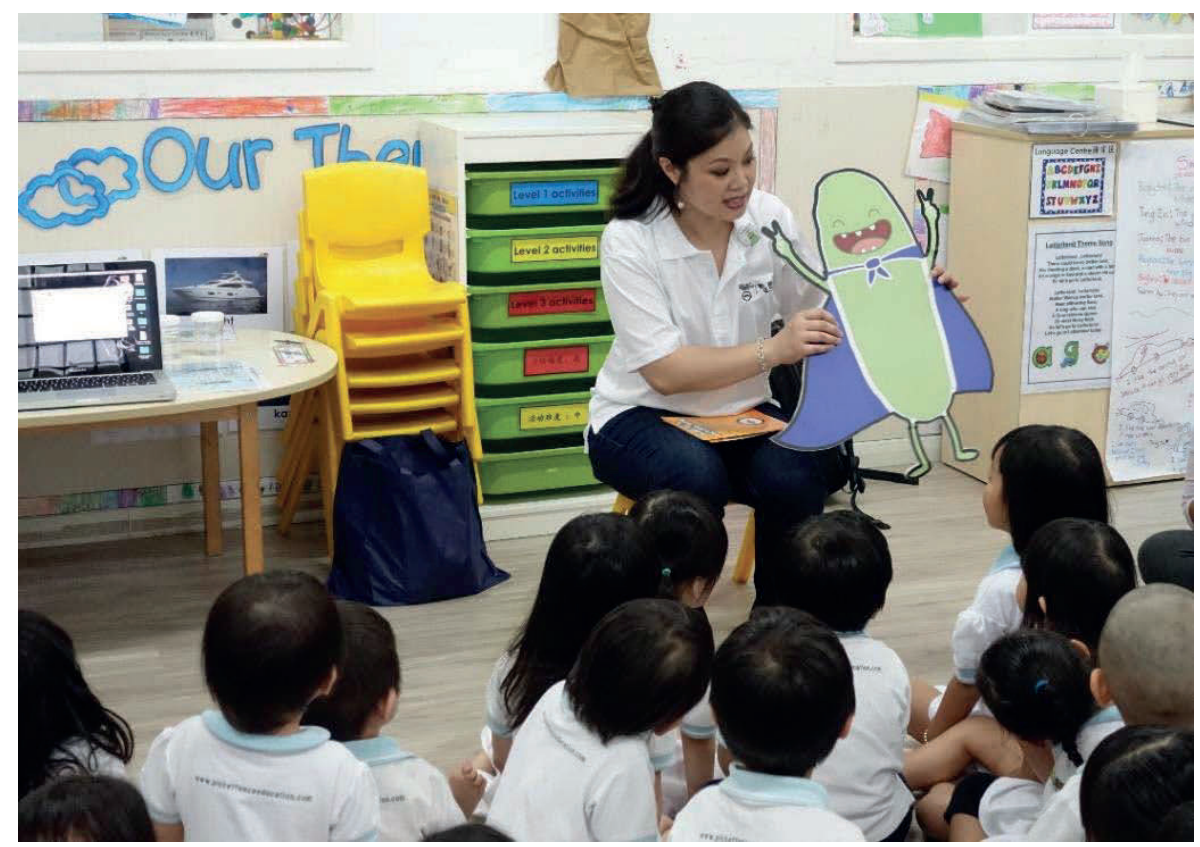

Figure 3. A Project Wolbachia - Singapore show-and-tell session for young children at a childcare centre.

Such citizen science initiatives have increased scientific understanding, and, by allowing participants to contribute directly, have helped build acceptance and a sense of ownership for the project.

\subsection{Comprehensive and Inclusive}

Given the need to reach out to people with diverse backgrounds, needs, and concerns, we set out to make our engagement programme as comprehensive and inclusive as possible. To reach a greater number of target groups, we employed multiple tiers of communication strategies, with varying levels of detail and engagement. 
Informational brochures, distributed to all residents and stakeholders at study/ release sites (Fig. 4), are used to convey short, key messages explaining the Wolbachia-Aedes technology and addressing what residents can do to help keep mosquito populations low.

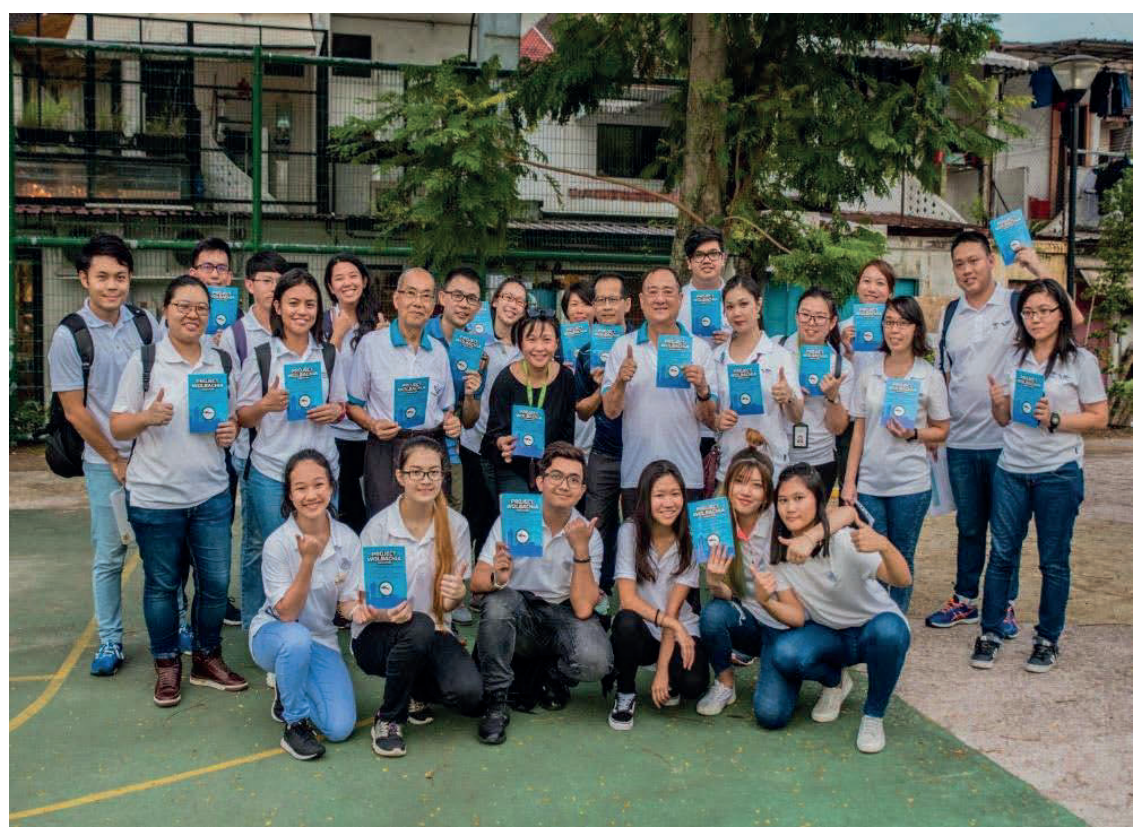

Figure 4. Informational brochures for distribution to residents and all stakeholders at the study/release sites.

These brochures use accessible language and infographics, and, given Singapore's multicultural landscape, are available in English, Mandarin, Malay and Tamil - the country's four official languages (Fig. 5).

More detailed information on the technology, releases, and trial results was made available through media releases (NEA 2018a, b, 2019c, d, e) and features in mainstream media (Co 2019; The Straits Times 2019), as well as via social media and other public communication channels (e.g. documentary features) (National Geographic Asia 2019). Members of the public interested in learning more can also visit NEA's Wolbachia website (NEA 2019a) for technical details, including scientific literature.

Besides disseminating information, we also conduct numerous face-to-face engagement activities, to engage residents more thoroughly and better understand their concerns. These activities include educational sessions in schools and preschools, door-to-door house visits, coffee chat sessions, apartment block and garden parties, roadshows in locations with heavy footfall (e.g. marketplaces, community centres, and shopping malls) (Fig. 6), and mosquito production facility tours. 


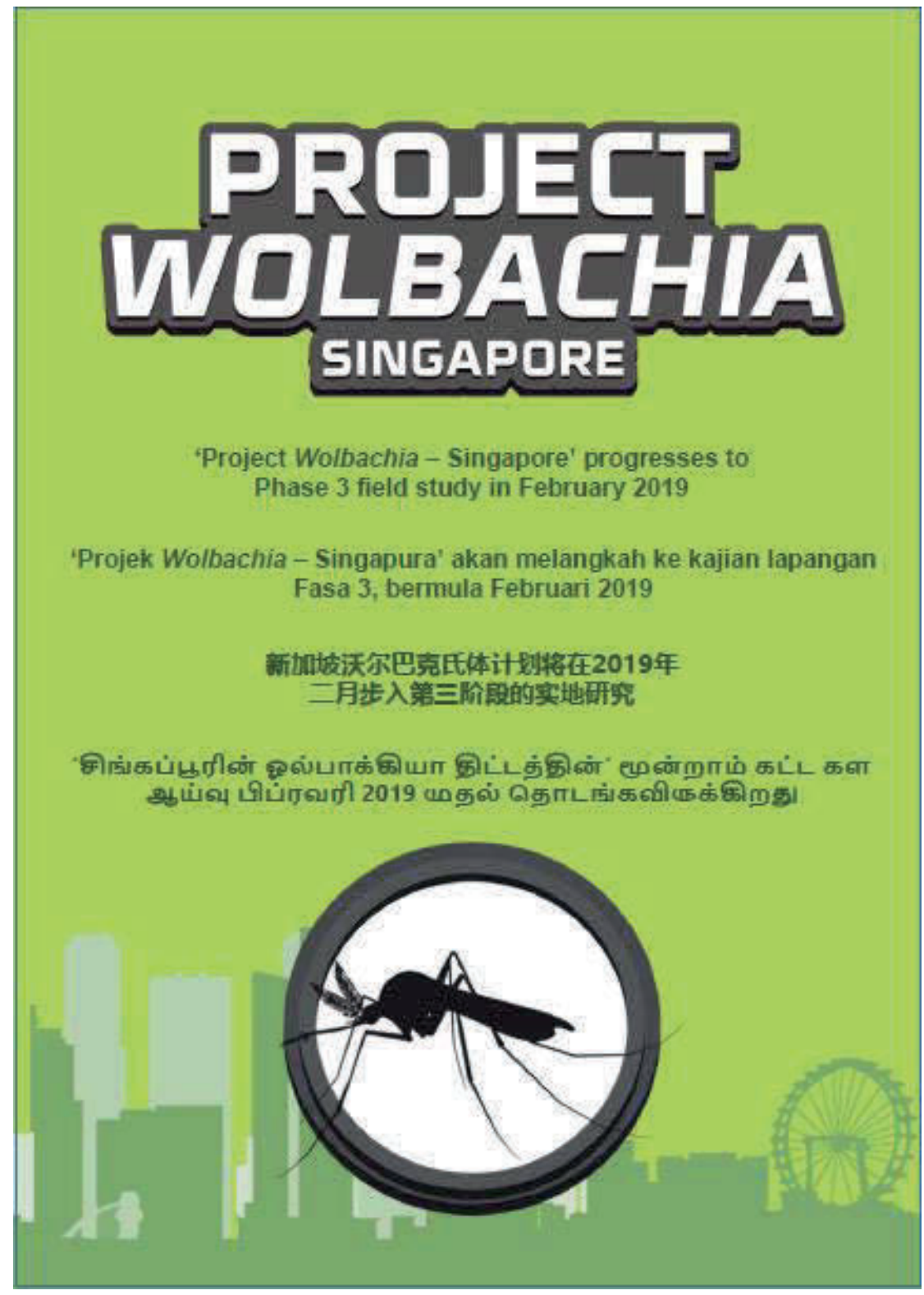

Figure 5. A brochure for Phase 3 of Project Wolbachia-Singapore, with information translated into Singapore's four official languages: English, Mandarin, Malay and Tamil. 

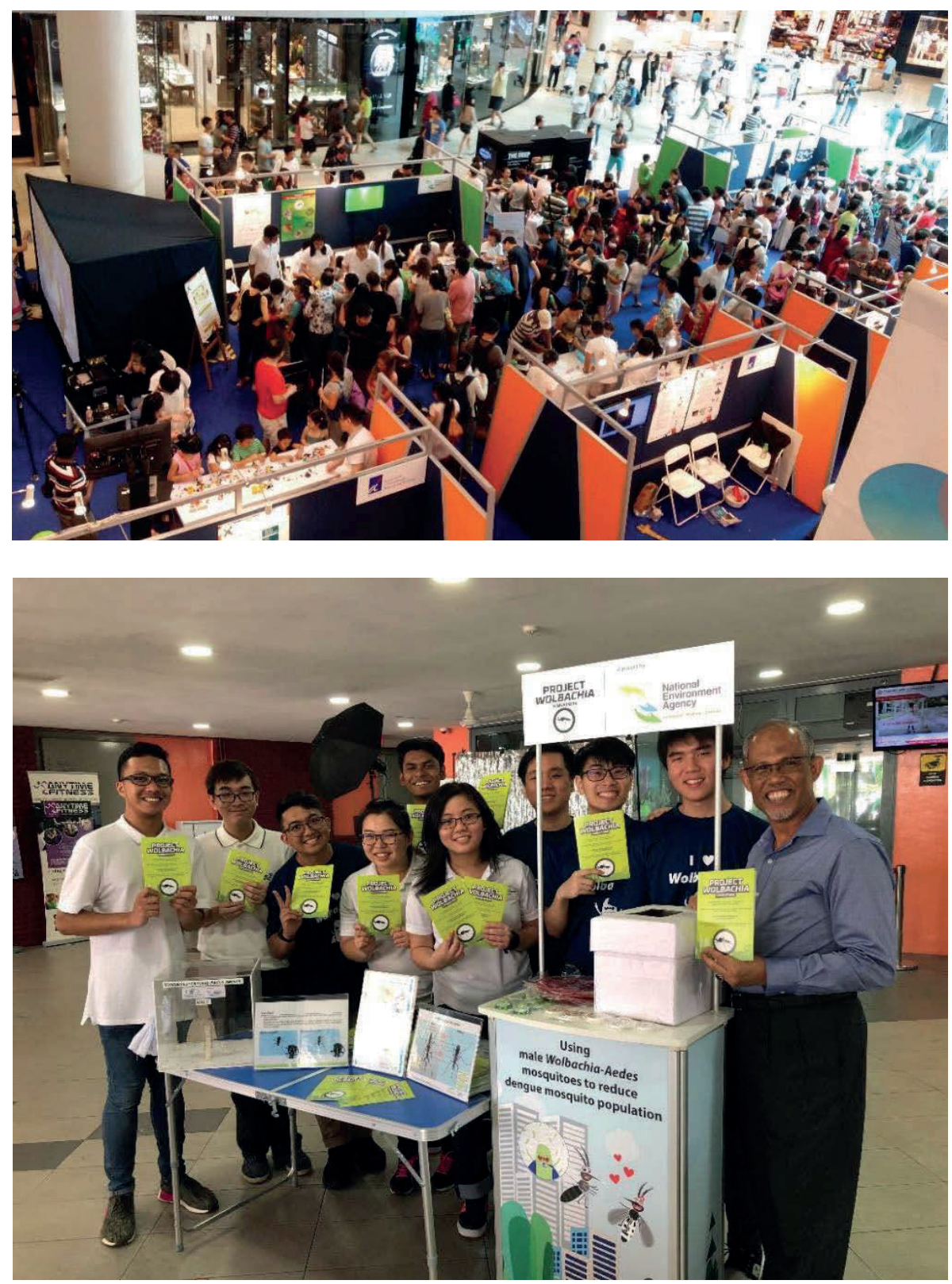

Figure 6. Outreach booths at public events/roadshows to showcase Project WolbachiaSingapore. Top: At a shopping mall. Bottom: At a community centre, with Singapore's Minister for the Environment and Water Resources, Mr. Masagos Zulkifli (far right). 
At the time of writing, we have held more than 100 public engagement sessions to raise awareness, answer queries, and solicit feedback about Project Wolbachia Singapore. To further extend the reach of our message, we engaged members of the public who are interested to help out as NEA Dengue Prevention Volunteers (DPVs), and who can then help to convey accurate information on Wolbachia-Aedes technology to their own networks within the community.

Leveraging the increasing pervasiveness of digital media, we used websites, social media platforms, and videos (NEA 2019f, g, h) to complement our face-to-face outreach activities and increase exposure. As Project Wolbachia - Singapore scales up and the number of affected members of the public involved in the project increases, we foresee digital media playing an even larger role in our community engagement strategy.

In developing activities to engage different groups and individuals, we relied strongly on our own local knowledge and experience gained, as well as that of leaders and residents in the community. At the same time, over the course of our outreach, we also learned through experience which engagement methods are the most effective and adjusted our strategy accordingly. For example, after one dialogue session with poor attendance, we shifted our strategy towards bringing our roadshows to where people are, rather than getting residents to go to a specific dialogue.

\subsection{Consultative and Responsive}

To promote Project Wolbachia - Singapore, an important scientific initiative that may result in tangible public health benefits, we adopted a consultative approach early on to respect the concerns and opinions of the public, taking seriously any feedback received from residents, members of the public, experts, and other stakeholders. Valid concerns (such as safety of the technology, potential negative ecological impacts, niche replacement by other mosquitoes, and unintentional release of female mosquitoes) were channelled into our risk assessment track, a parallel effort that has resulted in the publication of a risk assessment of the technology ( $\mathrm{Ng}$ et al. 2017; NEA 2019g).

Through house visits, roadshows, and dialogue sessions, we actively solicited feedback from residents and the public. We also consulted community or grassroots leaders, who have strong networks on the ground and a thorough understanding of residents' concerns. Following detailed engagement sessions to explain WolbachiaAedes technology and understand and address concerns, we were able to obtain leaders' support, approval and advocacy. The spectrum of views and concerns we received informed our release and engagement strategies later on.

We encouraged the community to learn more about the project and have also established mechanisms for the public to pose queries, voice concerns, and report incidents related to Project Wolbachia - Singapore. Feedback can be submitted via email, telephone hotline, an online reporting system, or verbally to our field officers. Our site managers also carry a dedicated mobile phone by which residents can reach them. 
Our officers follow up on feedback received by contacting each case through house visits, telephone calls, or email, to learn more about and address their individual situations. During such follow-ups, we engage the respondents with information about Project Wolbachia - Singapore, as well as attempt to address the issues raised. For example, a few residents who reported increased irritation from male mosquitoes were advised to keep their windows and doors closed, or to kill the mosquitoes as they normally would. If residents report bites, field officers are typically deployed to inspect the area for mosquito breeding sites.

Whilst these mosquitoes resulting from local breeding sites are unrelated to the release of male Wolbachia-Aedes mosquitoes, such feedback from residents has enabled us to find and eliminate mosquito breeding sites, an outcome that is consistent with the aims of Project Wolbachia - Singapore.

By respectfully engaging dissenting views and responding promptly to public feedback, we hope to improve the ability of the public to make informed opinions, thereby enhancing acceptance of Project Wolbachia - Singapore.

\section{IMPORTANCE OF SITUATIONAL AWARENESS}

Like most field studies, trials of Wolbachia-Aedes technology may be affected by environmental factors such as the presence of mosquito breeding habitats, temperature and rainfall variations, and imported mosquito-borne infections. Proper situational awareness is therefore important for community engagement efforts to swiftly address concerns from the public when they arise.

For example, a coincidental rise in the populations of other mosquito species (such as Culex quinquefasciatus Say) in the community, and a resulting increase in bites experienced, could lead to public doubts concerning Project Wolbachia - Singapore. In such cases, data on the population trends of the other mosquito species, together with an understanding of their biting behaviour and the detection of their breeding locations on site, could provide the evidence needed to be able to dissociate these experiences from the project.

Dengue cases may also occur within study sites, complicating the messaging that Project Wolbachia - Singapore suppresses the dengue-transmitting mosquito population and hence potentially also the risk of disease transmission. A good analysis of the situation within and around each of these sites is thus critical, as most of these reported dengue cases may have resulted from infections outside the release sites perhaps at school or at work - but were tagged to residential addresses within the sites.

Thus, our community engagement team is working closely with field officers to gather and respond to all information received on mosquito populations, mosquito sightings, and dengue cases within and around study sites, with the aim of assessing the cause of all issues raised by the community. 


\section{FUTURE DIRECTIONS AND CONCLUSION}

Thanks to early and comprehensive community engagement, nationwide online and face-to-face street surveys conducted in 2016 (prior to the launch of Project Wolbachia - Singapore) found high levels of acceptance for Wolbachia-Aedes technology, with a high number of respondents indicating that they had no concerns with the release of male Wolbachia-Aedes mosquitoes in their neighbourhoods. As Project Wolbachia - Singapore scales up (NEA 2019c), an ongoing challenge will be to expand community engagement accordingly, whilst still maintaining its quality. Whilst face-to-face interactions will remain a mainstay of our engagement strategy, this is highly labour-intensive; in the future, engagement through digital and social media will be scaled up to reach larger segments of the population. To maintain quality and impact, it will be critical to continually evaluate our community education and engagement strategies, and to modify them when necessary (Jayawardene et al. 2011; Healy et al. 2014). To this end, Project Wolbachia - Singapore as a whole, including the community engagement component, is regularly reviewed by an external panel comprising local and international experts (NEA 2019c).

We will also continue to conduct population surveys to ascertain public awareness, perceptions and attitudes towards Project Wolbachia - Singapore, which will allow us to further fine-tune our outreach and deployment strategies. Prior to the release of male Wolbachia-Aedes mosquitoes, a nationwide online survey conducted in 2016 revealed that $94 \%$ of the population had no objections to the release of male Wolbachia-Aedes mosquitoes in their neighbourhoods, and a face-to-face street survey targeting older respondents (aged 40 years and above) revealed similar results $-89 \%$ had no objections to such releases in their neighbourhoods, and $31 \%$ had heard of the project through mainstream media. During the Phase 1 field study in 2016, a survey conducted showed that more than $70 \%$ of the households interviewed had heard of our project, and more than $90 \%$ had no concerns with the releases.

In summary, community engagement is increasingly recognised as a critical dimension of biomedical and global health research, as well as the social sciences, especially where novel technologies such as Wolbachia-based control methods are concerned. We believe that respectful, impactful community engagement is crucial for the success of Wolbachia-based technologies and is recognised by senior leadership as a key project priority.

\section{ACKNOWLEDGEMENTS}

We work closely with the Singapore NEA's Corporate Communications Department and the 3P (People, Public and Private) Network Division, and are grateful for their advice as well as communications and engagement support. We are also grateful to the Residents' Committees and Citizens' Consultative Committees at the release sites, for their support and for assistance with outreach activities. Special thanks to Tampines Town Council, Yishun Town Council, Tampines West Community Centre, and Nee Soon East Community Centre for help with publicity and outreach. 


\section{REFERENCES}

Bhatt, S., P. W. Gething, O. J. Brady, J. P. Messina, A. W. Farlow, C. L. Moyes, J. M. Drake, J. S. Brownstein, A. G. Hoen, O. Sankoh, M. F. Myers, D. B. George, T. Jaenisch, G. R. Wint, C. P. Simmons, T. W. Scott, J. J. Farrar, and S. I. Hay. 2013. The global distribution and burden of dengue. Nature 496: 504-507.

Bourtzis, K., R. S. Lees, J. Hendrichs, and M. J. B. Vreysen. 2016. More than one rabbit out of the hat: radiation, transgenic and symbiont-based approaches for sustainable management of mosquito and tsetse fly populations. Acta Tropica 157: 115-130.

Co, C. 2019. New facility to produce up to 5 million Wolbachia mosquitoes weekly in fight against dengue. Channel News Asia, 2 December 2019.

Dyck, V. A., J. Hendrichs, and A. S. Robinson (eds.). 2021. Sterile Insect Technique - Principles and practice in Area-Wide Integrated Pest Management. Second Edition. CRC Press, Boca Raton, Florida, USA. 1200 pp.

Dieng, H., R. G. Saifur, A. H. Ahmad, M. C. Salmah, A. T. Aziz, T. Satho, F. Miake, Z. Jaal, S. Abubakar, and R. E. Morales. 2012. Unusual developing sites of dengue vectors and potential epidemiological implications. Asian Pacific Journal of Tropical Biomedicine 2: 228-232.

Ernst, K. C., S. Haenchen, K. Dickinson, M. S. Doyle, K. Walker, A. J. Monaghan, and M. H. Hayden. 2015. Awareness and support of release of genetically modified "sterile" mosquitoes, Key West, Florida, USA. Emerging Infectious Diseases 21(2): 320-324.

Hapuarachchi, H. C., C. Koo, J. Rajarethinam, C.-S. Chong, C. Lin, G. Yap, L. Liu, Y.-L. Lai, P. L. Ooi, J. Cutter, and L. C. Ng. 2016. Epidemic resurgence of dengue fever in Singapore in 2013-2014: A virological and entomological perspective. BMC Infectious Diseases 16: 300.

Healy, K., G. Hamilton, T. Crepeau, S. Healy, I. Unlu, A. Farajollahi, and D. M. Fonseca. 2014. Integrating the public in mosquito management: Active education by community peers can lead to significant reduction in peridomestic container mosquito habitats. PLoS One 9(9): e108504.

Jayawardene, W., D. K. Lohrmann, A. H. Youssefagha, and D. C. Nilwala. 2011. Prevention of dengue fever: An exploratory school-community intervention involving students empowered as change agents. The Journal of School Health 81: 566-573.

Kolopack, P. A., J. A. Parsons, and J. V. Lavery. 2015. What makes community engagement effective? Lessons from the Eliminate Dengue program in Queensland Australia. PLoS Neglected Tropical Diseases 9(4): e0003713.

Lee, K.-S., Y.-L. Lai, S. Lo, T. Barkham, P. Aw, P.-L. Ooi, J. C. Tai, M. Hibberd, P. Johansson, S. P. Khoo, and L. C. Ng. 2010. Dengue virus surveillance for early warning, Singapore. Emerging Infectious Diseases 16: 847-849.

Lees, R. S., J. R. L. Gilles, J. Hendrichs, M. J. B. Vreysen, and K. Bourtzis. 2015. Back to the future: The sterile insect technique against mosquito disease vectors. Current Opinion in Insect Science 10: $156-162$.

Lees, R. S., D. O. Carvalho, and J. Bouyer. 2021. Potential impact of integrating the Sterile Insect Technique into the fight against disease-transmitting mosquitoes, pp. 1081-1118. In V. A. Dyck, J. Hendrichs, and A. S. Robinson (eds.), Sterile Insect Technique - Principles and practice in Area-wide Integrated Pest Management. Second Edition. CRC Press, Boca Raton, Florida, USA.

Liew, C., and L. C. Ng. 2017. The buzz behind Project Wolbachia - Singapore. ENVISION Magazine 13 $82-83$.

Low, S.-L., S. Lam, W.-Y. Wong, D. Teo, L. C. Ng, and L.-K. Tan. 2015. Dengue seroprevalence of healthy adults in Singapore: Serosurvey among blood donors, 2009. American Journal of Tropical Medicine and Hygiene 93: 40-45.

McMeniman, C. J., R. V. Lane, B. N. Cass, A. W. Fong, M. Sidhu, Y.-F. Wang, and S. L. O'Neill. 2009. Stable introduction of a life-shortening Wolbachia infection into the mosquito Aedes aegypti. Science 323: 141-144.

McNaughton, D., and T. T. H. Duong. 2014. Designing a community engagement framework for a new dengue control method: A case study from central Vietnam. PLoS Neglected Tropical Diseases 8(5): e2794.

National Geographic Asia. 2019. City of innovation: Singapore. Full Documentary.

(NEA) National Environment Agency, Singapore. 2018a. Objectives met for Phase 1 of Wolbachia field studies. NEA to commence Phase 2 Wolbachia-Aedes field study at three sites in April 2018. 
(NEA) National Environment Agency, Singapore. 2018b. Project Wolbachia - Singapore welcomes new collaborator for ongoing Phase 2 field study.

(NEA) National Environment Agency, Singapore. 2019a. Project Wolbachia Singapore. WolbachiaAedes mosquito suppression strategy.

(NEA) National Environment Agency, Singapore. 2019b. Prevent Aedes mosquito breeding.

(NEA) National Environment Agency, Singapore. 2019c. Project Wolbachia - Singapore progresses to Phase 3 field study at two extended sites in February 2019.

(NEA) National Environment Agency, Singapore. 2019d. Successful study paves the way for doubling the size of male Wolbachia-Aedes mosquito release.

(NEA) National Environment Agency, Singapore. 2019e. New NEA facility to boost production of male Wolbachia-Aedes aegypti mosquitoes to benefit more residents.

(NEA) National Environment Agency, Singapore. 2019f. Wolbachia video series. Part I: Safety and efficacy studies; Part II: Behind the scenes; Part III: Engaging stakeholders and the community; Part IV: Small-scale field study; Part V: Project progresses to Phase 2 field study.

(NEA) National Environment Agency, Singapore. 2019g. Wolbachia in my neighbourhood.

(NEA) National Environment Agency, Singapore. 2019h. Wolbachia is SAFE.

Ng, L. C., C. Liew, R. Gutierrez, C. S. Chong, C. H. Tan, G. Yap, J. P. S. Wong, and I. M. Li. 2017. How safe is Wolbachia for Aedes control? A risk assessment for the use of male Wolbachia-carrying Aedes aegypti for suppression of the Aedes aegypti mosquito population. Epidemiological News Bulletin 43: 8-16.

Ong, J., X. Liu, J. Rajarethinam, G. Yap, D. Ho, and L. C. Ng. 2019. A novel entomological index, Aedes aegypti breeding percentage, reveals the geographical spread of the dengue vector in Singapore and serves as a spatial risk indicator for dengue. Parasites \& Vectors 12: 17.

(SingStat) Singapore Department of Statistics. 2019. Households - Latest Data 2018.

Subramaniam, T. S. S., H. L. Lee, N. W. Ahmad, and S. Murad. 2012. Genetically modified mosquito: The Malaysian public engagement experience. Biotechnology Journal 7: 1323-1327.

The Straits Times. 2019. NEA to test releasing mosquitoes via drone as Project Wolbachia moves to Phase 4 of fight against dengue. 7 October 2019. 